\title{
INVESTIGAÇÃO DE DOENÇA ATEROSCLERÓTICA EM PACIENTES TRANSPLANTADOS RENAIS E EM LISTA DE ESPERA EM ACOMPANHAMENTO AMBULATORIAL POR MEIO DE ECOGRAFIA DE ARTÉRIAS CARÓTIDAS
}

\author{
Investigation of atherosclerotic disease in renal transplanted recipients and on waiting list in \\ ambulatory monitoring through carotid artery ultrasound
}

\author{
Ângela Quatrin Campagnoloํ, Felipe da Silva Souza², Paulo Fernando Dotto Bau³, Mateus Diniz Marques³, \\ Alexandre Antônio Naujorks ${ }^{4}$, Henry Mor Pansard ${ }^{5}$, Fábio Prestes de Campos ${ }^{6}$
}

\begin{abstract}
RESUMO
O transplante renal é o tratamento de escolha para pacientes com doença renal crônica terminal. Atualmente, vem aumentando a proporção de óbitos relacionados às doenças cardiovasculares nesses pacientes, as quais são as principais manifestações da doença aterosclerótica. Objetivos: avaliar a prevalência de aterosclerose carotídea em pacientes transplantados renais e em lista de espera para o procedimento no Hospital Universitário de Santa Maria por meio de ecografia de artérias carótidas e correlacionar sua presença com o escore de Framingham. Métodos: Estudo transversal, prospectivo, avaliou 59 pacientes transplantados renais e não transplantados que aguardavam em lista de espera para transplante renal do ambulatório de Transplante Renal do Hospital Universitário de Santa Maria entre janeiro de 2012 a março de 2013. Realizou-se ecografia de artérias carótidas para diagnóstico e quantificação de aterosclerose carotídea, bem como cálculo do escore de Framingham por meio das variáveis coletadas. Resultados: A prevalência de placas carotídeas foi de $59,38 \%$ nos pacientes submetidos ao transplante renal e de 70,37\% naqueles em lista de espera. Não houve associação significativa entre os grupos quanto à presença de placas carotídeas $(\mathrm{p}=0,379)$ ou de sua gravidade $(\mathrm{p}=0,704)$. O grupo submetido a transplante renal esteve maior tempo em terapia dialítica $(55,25 \pm 44,16$ meses vs. $28,15 \pm 36,50$ meses, $\mathrm{p}=0,00079)$, tinha menor média de idade $(45,09 \pm 13,04$ vs $52,48 \pm$ $14,18$ anos, $\mathrm{p}=0,042)$, menor número de pacientes diabéticos ( $9 \%$ vs 52\%, $\mathrm{p}=0,00033)$ e menor escore de Framingham $(8,72 \% \pm$ $7,5$ vs. $16,51 \% \pm 11,97, p=0,002)$. Não houve diferença significativa entre a presença de placa carotídea e o tempo de transplante renal $(\mathrm{p}=0,399)$ ou tipo de esquema imunossupressor $(\mathrm{p}=0,939)$. Encontrou-se correlação intermediária (coef. Spearman $=0,47, \mathrm{p}=0,0065)$ entre o grau de Framingham e a gravidade da placa carotídea nos pacientes submetidos a transplante renal. $\mathrm{Na}$ análise de regressão logística para fatores associados à presença/ausência de placa carotídea, encontrou-se associação entre o Escore de Framingham e chance de placa carotídea (OR=1,104 [1,008-1,210, IC OR 95\%], p=0,033). Conclusão: A doença aterosclerótica carotídea apresenta prevalência elevada na população estudada. Os fatores de risco cardiovasculares tradicionais, utilizados no escore de Framingham têm um papel importante no desenvolvimento das placas carotídeas.
\end{abstract}

Descritores: Transplante Renal; Aterosclerose; Artérias Carótidas.

Instituição:

${ }^{1}$ Programa de Residência Médica em Cardiologia do

${ }^{2}$ Programa de Residência Médica em Ecocardiografia do Hospital

Universitário de Santa Maria - Santa Maria/RS, Brasil.

${ }^{3}$ Departamento de Clinica Médica da Universidade Federal de Santa Maria - Santa Maria/RS, Brasil.

${ }^{4}$ Serviço de Cardiologia do Hospital Universitário de Santa Maria -

Santa Maria/RS, Brasil.

${ }^{5}$ Serviço de Nefrologia do Hospital Universitário de Santa Maria -

Santa Maria/RS, Brasil.

${ }^{6}$ Programa de Residência Médica em Nefrologia do Hospital

Universitário de Santa Maria - Santa Maria/RS, Brasil.

\section{Correspondência:}

Ângela Quatrin Campagnolo

Depto.Clínica Médica (CCS) - Campus da UFSM - Av. Roraima, 1000, s/1337, prédio 26, CEP 97105-900, Santa Maria/RS

Tel.: (55) 9947-1847 - E-mail: angelaqcampagnolo@gmail.com

Recebido em: 30/04/2013

Aceito em: 28/06/3013

\section{INTRODUÇÃO}

O transplante renal é o trata mento de escolha para pacientes com doença renal crônica terminal, trazendo sobrevida e qualidade de vida superiores à terapia dialítica. ${ }^{1}$

Durante o ano de 2012, o número absoluto de transplantes renais no Brasil foi de 5385, sendo 1488 de doadores vivos e 3897 de doadores falecidos. Esses dados equivalem, por milhão da população (PMP), a 28,2 transplantes renais, sendo 7,8 pmp de doadores vivos e 20,4 de doadores falecidos. O Rio Grande do Sul foi o primeiro estado em número de transplantes renais PMP durante o ano de 2012, perfazendo um total de 51,2 transplantes PMP. ${ }^{2}$

Receptores de transplante renal apresentam elevada prevalência de doença aterosclerótica. Os fatores de risco cardiovascular nessa população são múltiplos e interrelacionados, sendo as complicações cardiovasculares a 
Ângela Quatrin Campagnolo, Felipe da Silva Souza, Paulo Fernando Dotto Bau, Mateus Diniz Marques, Alexandre Antônio Naujorks, Henry Mor Pansard, Fábio Prestes de Campos

principal causa de óbito em longo prazo em pacientes com o funcionamento adequado do enxerto. ${ }^{3}$ Estudos prévios indicam elevada prevalência de placas ateroscleróticas carotídeas e um aumento da espessura médio-intimal em receptores de transplante renal, alterações que parecem ser fatores de risco independentes de óbito em longo prazo. ${ }^{4,5}$

É fundamental no transplante de órgãos a prevenção da rejeição ao órgão recebido. Assim, o uso de drogas imunossupressoras é a chave do sucesso na manutenção da função do enxerto. ${ }^{6}$ Porém, algumas medicações dessa terapia imunossupressora exacerbam fatores de risco tradicionais para aterosclerose como a dislipidemia, hipertensão, hiperglicemia e hiperhomocisteinemia. ${ }^{7}$

O Estudo Cardíaco de Framingham demonstrou que certos fatores de risco estão intimamente relacionados ao desenvolvimento de doença arterial coronariana. ${ }^{8}$

Desse estudo, originou-se o escore de risco de Framingham, que estima o risco de homens e mulheres sem diagnóstico prévio de aterosclerose terem eventos cardiovasculares do tipo infarto do miocárdio ou óbito, em 10 anos. Assim, ele auxilia a identificar adultos de alto risco para doença arterial coronariana, os quais se beneficiam de determinada estratégia preventiva terapêutica. ${ }^{9,10}$

A presença de doença aterosclerótica em qualquer território vascular é reconhecida como um significativo aumento na probabilidade de doença cardiovascular em outros territórios vasculares. A presença de doença vascular aterosclerótica não coronariana é considerada como um equivalente de risco para evento cardiovascular futuro. ${ }^{11}$

A ultrassonografia de alta resolução modo-B é um método útil e não invasivo para o diagnóstico de aterosclerose carotídea. ${ }^{12}$ Esse método é seguro e com custo acessível; possui sensibilidade entre $86-90 \%$ e especificidade de 87 $94 \%$ em detectar estenose significativa (maior ou igual a $70 \%)$ na artéria carótida interna. ${ }^{13}$

Apesar da redução da mortalidade nos pacientes submetidos ao transplante renal nos últimos anos, está aumentando a proporção de óbitos relacionados às doenças cardiovasculares nesses pacientes, as quais são as principais manifestações da doença aterosclerótica. ${ }^{5}$ Este estudo tem como objetivo avaliar a prevalência de aterosclerose carotídea em pacientes transplantados renais e em lista de espera para o procedimento em acompanhamento ambulatorial no Hospital Universitário de Santa Maria por meio de ecografia de artérias carótidas e correlacionar sua presença com o escore de Framingham.

\section{PACIENTES E MÉTODOS}

Esse estudo de delineamento transversal foi realizado durante os meses de janeiro de 2012 a março de 2013. Foram convidados a participar do estudo pacientes com mais de 18 anos, com diagnóstico de doença renal crônica terminal (a doença renal crônica tratada com diálise ou com transplante renal), ${ }^{14}$ transplantados renais e não transplantados que aguardavam em lista de espera para transplante renal e que frequentaram o ambulatório de Transplante Renal do Hospital Universitário de Santa Maria, localizado na cidade de Santa Maria, no estado do Rio Grande do Sul. A amostragem foi realizada por conveniência, e os pacientes que atendiam aos critérios de elegibilidade eram convidados a participar da pesquisa, conforme sua disponibilidade. Foram excluídos do estudo pacientes com evidência de doença cardiovascular (doença arterial coronariana, insuficiência cardíaca, doença cerebrovascular e doença arterial periférica). A coleta dos dados foi realizada sempre pelo mesmo pesquisador.

A pesquisa foi realizada conforme as normas da Resolução 196/96 do Conselho Nacional de Saúde, que dispõe sobre pesquisa com seres humanos. O projeto foi aprovado pelo Comitê de Ética de Pesquisa da Universidade Federal de Santa Maria. Todos os pacientes incluídos receberam e prestaram consentimento escrito através do Termo de Consentimento Livre e Esclarecido.

Os pacientes foram submetidos a um questionário específico direcionado às seguintes variáveis demográficas: sexo, idade, tipo de terapia dialítica e tempo, medicamentos em uso, patologias prévias e atuais em tratamento. Enfatizou-se sintomas e diagnósticos cardiovasculares, tabagismo atual, realização de transplante renal, tempo e tipo de doador. Coletaram-se durante a consulta o valor da pressão arterial, o peso e a estatura, bem como os resultados dos exames laboratoriais de colesterol total e frações, triglicerídeos e glicemia de jejum. Realizou-se estudo ultrassonográfico arterial carotídeo, empregando-se transdutor linear de 7,5 a $10 \mathrm{MHz}$, no aparelho Esaote no setor de métodos gráficos do Hospital Universitário de Santa Maria. Empregaramse técnicas ecográficas de modo bidimensional, Doppler pulsado, Doppler com mapeamento de fluxo a cores e power Doppler. A estratificação do grau de estenose da artéria carótida interna é realizada com base na escala de cinza e ecografia por Doppler e os resultados são expressos nas seguintes faixas: normal (sem estenose), estenose menor do que $50 \%$, estenose entre $50-69 \%$ e estenose maior ou igual a $70 \%$, próxima à oclusão e oclusão total. ${ }^{15}$

A artéria carótida interna é considerada normal quando o pico sistólico de velocidade é menor do que $125 \mathrm{~cm} / \mathrm{s}$ e nenhuma placa ou espessamento intimal for visível à ecografia. A estenose menor do que $50 \%$ é diagnosticada por um pico de velocidade sistólico menor do que $125 \mathrm{~cm} / \mathrm{s}$ e placa ou espessamento intimal for visível. A estenose entre $50-69 \%$ é diagnosticada quando a velocidade de pico sistólico está entre $125-230 \mathrm{~cm} / \mathrm{s}$ e a placa for visível. A estenose maior do que $70 \%$, porém menor do que próximo à oclusão, é diagnosticada quando a velocidade de pico sistólico for maior do que $230 \mathrm{~cm} / \mathrm{s}$, a placa for visualizada 
e também houver um estreitamento luminal visto em escala de cinza e à ecografia por Doppler colorido. Nos casos de estenose próxima da oclusão, os parâmetros de velocidade não podem ser aplicados, pois esta pode ser elevada, baixa ou indetectável, assim, o diagnóstico é estabelecido primariamente por um lúmen arterial marcadamente diminuído no Doppler colorido. Deve haver suspeita de oclusão total da artéria carótida interna quando não houver lúmen patente detectável na escala de cinza da ecografia e também não for detectado fluxo com Doppler potente, colorido e espectral. ${ }^{15}$

Todas as ecografias carotídeas foram feitas pelo mesmo operador. Consultou-se no prontuário o resultado do exame e registrou-se no protocolo de coleta de dados. A presença de placa carotídea foi definida como um espessamento da camada médio-intimal maior do que $1,5 \mathrm{~mm} \cdot{ }^{12}$

Foram considerados portadores de hipertensão, os pacientes que utilizavam medicações anti-hipertensivas ${ }^{10}$ e/ou que tinham pressão arterial maior ou igual do que $140 / 90 \mathrm{mmHg}$ na consulta da coleta de dados e em algum outro registro do prontuário. ${ }^{16}$

O diagnóstico de diabetes foi considerado nos pacientes que estavam em uso de medicação específica (hipoglicemiantes orais ou insulina) e/ou que tinham glicemia de jejum maior ou igual a $126 \mathrm{mg} / \mathrm{dl}^{10}$ na consulta da coleta dos dados e em algum outro registro de exame prévio do prontuário.

Foram definidos dislipidêmicos os pacientes em uso de estatinas ou fibratos, ou com um ou mais dos valores alterados: CT $>200 \mathrm{mg} / \mathrm{dL}$; LDL $>160 \mathrm{mg} / \mathrm{dL}, \quad \mathrm{TG}>$ $150 \mathrm{mg} / \mathrm{dL}, \mathrm{HDL}<40 \mathrm{mg} / \mathrm{dL}$ em homens e $<50 \mathrm{mg} / \mathrm{dL}$ em mulheres. ${ }^{9} \mathrm{O}$ critério para tabagismo foi ter fumado pelo menos um cigarro no último mês, conforme auto relato do paciente. ${ }^{10}$

A análise estatística foi realizada com auxílio do software Statistic versão 9.1. As variáveis qualitativas foram representadas por porcentagens e analisadas pelo teste de qui-quadrado. As variáveis quantitativas foram representadas pelas médias \pm desvios padrões ou medianas e tiveram a normalidade das suas distribuições verificadas pelo teste de Shapiro Wilk com nível de significância de 5\%.

Para as variáveis contínuas de distribuição normal, foi realizado o teste paramétrico $\mathrm{T}$ de Student e para as variáveis contínuas de distribuição anormal, foi realizado o teste não paramétrico $U$ de Mann-Whitney para comparação das mesmas.

Realizou-se o teste de correlação de Spearman para avaliar a correlação entre o grau de Framingham e a gravidade da placa carotídea e análise de regressão logística para fatores associados à presença/ausência de placa carotídea. Foram considerados significativos resultados de $\mathrm{p}<0,05$.

\section{RESULTADOS}

Um total de 59 pacientes portadores de doença renal crônica terminal foi incluído no estudo, sendo 32 já submetidos a transplante renal. Suas características clínicas e demográficas estão sumarizadas na Tabela 1 .

A média de idade do grupo que realizou o transplante renal foi de 45,09 $\pm 13,04$ anos e do grupo que não realizou o transplante foi de $52,48 \pm 14,18$ anos. O grupo dos não transplantados tem maior média de idade do que o grupo de pacientes submetidos a transplante renal $(\mathrm{p}=0,042)$.

O tempo médio de diálise foi de 55,25 $\pm 44,16$ meses no grupo do transplante renal e de $28,15 \pm 36,50$ meses no outro grupo. O grupo submetido ao transplante renal esteve maior tempo em terapia dialítica $(\mathrm{p}=0,00079)$.

O grupo que recebeu o transplante renal possui maiores valores de HDL colesterol $(53,90 \pm 20,41, \mathrm{p}=0,045)$ e menores valores de glicemia de jejum $(95,03 \pm 16.80, p=0,00036)$. Nenhum paciente estava fumando no último mês durante a coleta dos dados.

Houve associação significativa entre os grupos em relação à presença de diabetes $(\mathrm{p}=0,00033)$. $\mathrm{O}$ grupo dos pacientes não submetidos ao transplante renal tem maior número de diabéticos $(52 \%)$ e maiores valores de glicemias de jejum $(149,92 \pm 109,62 \mathrm{p}=0,00036)$.

Não há associação significativa entre os grupos quanto ao sexo $(p=0,922)$, hipertensão arterial sistêmica $(p=0,522)$ e dislipidemia $(\mathrm{p}=0,321)$. A média dos índices de massa corporal $(p=0,406)$, os valores de colesterol total $(p=0,153)$, fração LDL de colesterol $(\mathrm{p}=0,0525)$ e triglicerídeos $(\mathrm{p}=0,0714)$ não diferem entre os grupos.

A comparação entre os grupos em relação ao escore de Framingham e quanto à presença e gravidade da placa carotídea estão apresentados na tabela 2.

O Escore de Framingham médio dos pacientes transplantados foi de $8,72 \pm 7,50$ e dos em lista de espera foi de $16,51 \pm 11,97$, diferindo significativamente $(p=0,002)$.

Houve associação significativa entre os grupos em relação ao grau de risco pelo escore de Framingham ( $\mathrm{p}=0,012$ ). Assim, o grupo dos pacientes em lista de espera para o transplante renal tem maior número de pacientes com alto risco no escore de Framingham.

No grupo de pacientes submetidos a transplante renal, encontrou-se uma prevalência de $59,38 \%$ de placas carotídeas. Entre os pacientes em lista de espera, encontrou-se uma prevalência de $70,37 \%$ de placas carotídeas. Não há associação significativa entre os grupos quanto à presença de placas carotídeas $(\mathrm{p}=0,379)$ ou de sua gravidade $(\mathrm{p}=0,704)$. 
Ângela Quatrin Campagnolo, Felipe da Silva Souza, Paulo Fernando Dotto Bau, Mateus Diniz Marques, Alexandre Antônio Naujorks, Henry Mor Pansard, Fábio Prestes de Campos

Tabela 1 - Características da amostra do grupo

\begin{tabular}{|c|c|c|c|}
\hline & $\underset{(n=32)}{\text { Grupo Tx }}$ & $\begin{array}{l}\text { Grupo não Tx } \\
(\mathrm{n}=27)\end{array}$ & $\mathrm{p}$ valor \\
\hline Sexo feminino ${ }^{1}$ & $15(47 \%)$ & $13(48 \%)$ & \multirow[t]{2}{*}{$0,922^{6}$} \\
\hline Sexo masculino ${ }^{1}$ & $17(53 \%)$ & $14(52 \%)$ & \\
\hline Idade $^{2}$ (anos) & $45,09 \pm 13,04$ & $52,48 \pm 14,18$ & $0,042^{4}$ \\
\hline HAS $^{1}$ & $28(88 \%)$ & $22(81 \%)$ & $0,522^{6}$ \\
\hline $\mathrm{DM}^{1}$ & $3(9 \%)$ & $14(52 \%)$ & \multirow[t]{2}{*}{$0,00033^{6}$} \\
\hline Tabagismo $^{1}$ & $0(0 \%)$ & $0(0 \%)$ & \\
\hline Dislipidemia $^{1}$ & $28(87 \%)$ & $21(78 \%)$ & $0,321^{6}$ \\
\hline $\begin{array}{l}\text { Tempo de diálise } \\
\text { (meses) }\end{array}$ & $55,25 \pm 44,16$ & $28,15 \pm 36,50$ & $0,00079^{5}$ \\
\hline $\mathrm{IMC}^{2}$ & $\begin{array}{c}41,00^{3} \\
25,90 \pm 4,27\end{array}$ & $\begin{array}{c}11,0^{3} \\
26,86 \pm 4,54\end{array}$ & $0,406^{4}$ \\
\hline $\mathrm{CT}(\mathrm{mg} / \mathrm{dL})^{2}$ & $181,81 \pm 40,34$ & $199,0 \pm 52,87$ & $0,153^{5}$ \\
\hline $\operatorname{HDL}(\mathrm{mg} / \mathrm{dL})^{2}$ & $\begin{array}{c}175,00^{3} \\
53,90 \pm 20,41\end{array}$ & $\begin{array}{c}190,00^{3} \\
43,18 \pm 11,86\end{array}$ & $0,045^{5}$ \\
\hline $\mathrm{LDL}(\mathrm{mg} / \mathrm{dL})^{2}$ & $\begin{array}{c}48,50^{3} \\
98,09 \pm 31,05\end{array}$ & $\begin{array}{c}43,19^{3} \\
119,33 \pm 45,20\end{array}$ & $0,0525^{5}$ \\
\hline $\mathrm{TG}(\mathrm{mg} / \mathrm{dL})^{2}$ & $\begin{array}{c}88,50^{3} \\
149,62 \pm 64,80\end{array}$ & $\begin{array}{c}106,00^{3} \\
197,77 \pm 109,82\end{array}$ & $0,0714^{5}$ \\
\hline $\mathrm{GJ}(\mathrm{mg} / \mathrm{dL})^{2}$ & $\begin{array}{c}132,00^{3} \\
95,03 \pm 16,80\end{array}$ & $\begin{array}{c}177,00^{3} \\
149,92 \pm 109,62\end{array}$ & \multirow[t]{2}{*}{$0,00036^{5}$} \\
\hline & $89,50^{3}$ & $113,00^{3}$ & \\
\hline
\end{tabular}

Tx $=$ Transplante renal

$\mathrm{IMC}=$ Índice de massa corporal $\rightarrow$ calculado pelo peso $(\mathrm{em} \mathrm{kg})$, dividido pela estatura (em metros) ao quadrado

$\mathrm{CT}=$ Colesterol total

HDL = Lipoproteína de alta densidade

LDL = Lipoproteína de baixa densidade

$\mathrm{GJ}=$ Glicemia de jejum

HAS = Hipertensão arterial sistêmica

$\mathrm{DM}=$ Diabetes

${ }^{1}$ Os dados são apresentados como número de indivíduos = n (\%), exceto quando descrito o contrário.

${ }^{2}$ Dados expressos em média \pm desvio padrão

${ }^{3}$ Mediana - representada nas variáveis não paramétricas

${ }^{4}$ Significância estatística estabelecida por meio do teste T de student

${ }^{5}$ Significância estatística estabelecida por meio do teste U de MannWhitney

${ }^{6}$ Significância estatística estabelecida pelo teste do qui-quadrado.

Realizou-se uma análise do subgrupo dos pacientes submetidos ao transplante renal em relação à presença de placa carotídea, a qual está representada na tabela 3 . O tempo médio após o transplante renal foi de 53,34 $\pm 59,59$ meses. Não houve diferença significativa entre o tempo de transplante renal e a presença de placa carotídea $(\mathrm{p}=0,399)$.

Em relação ao tipo de doador, $34 \%$ de nossa amostra receberam rim de doador vivo e $66 \%$ de doador falecido. Não houve associação significativa entre o tipo de doador e placa carotídea $(\mathrm{p}=0,722)$.

Os pacientes transplantados renais receberam três tipos
Tabela 2 - Escore de Framingham, presença e gravidade da placa carotídea por grupo

\begin{tabular}{|c|c|c|c|}
\hline & $\begin{array}{c}\text { Grupo Tx } \\
(n=32)\end{array}$ & $\begin{array}{l}\text { Grupo não Tx } \\
(\mathrm{n}=27)\end{array}$ & $\mathrm{p}$ valor \\
\hline Framingham $(\%)^{2}$ & $\begin{array}{c}8,72 \pm 7,50 \\
5,25^{3}\end{array}$ & $\begin{array}{c}16,51 \pm 11,97 \\
14,90^{3}\end{array}$ & $0,002^{4}$ \\
\hline Risco de Framingham ${ }^{1}$ & & & $0,012^{5}$ \\
\hline Baixo & $19(59,3 \%)$ & $9(33,3 \%)$ & \\
\hline Moderado & $12(37,5 \%)$ & $10(37 \%)$ & \\
\hline Alto & $1(3,1 \%)$ & $8(29,6 \%)$ & \\
\hline $\begin{array}{l}\text { Presença de placa } \\
\text { carotídea }^{1}\end{array}$ & $19(59,38 \%)$ & $19(70,37 \%)$ & $0,379^{5}$ \\
\hline Estenose carotídea $^{1}$ & & & $0,704^{5}$ \\
\hline $\begin{array}{c}\text { Leve } \\
(<50 \%)\end{array}$ & $15(78,95 \%)$ & $14(73,68 \%)$ & \\
\hline $\begin{array}{l}\text { Moderada } \\
50-69 \%)\end{array}$ & $4(21,05 \%)$ & $5(26,32 \%)$ & \\
\hline $\begin{array}{l}\text { Grave } \\
(\geq 70 \%)\end{array}$ & 0 & 0 & \\
\hline
\end{tabular}

$\mathrm{Tx}=$ Transplante renal

${ }^{1}$ Os dados são apresentados como número de indivíduos = n (\%), exceto quando descrito o contrário.

${ }^{2}$ Dados expressos em média \pm desvio padrão

${ }^{3}$ Mediana - representada nas variáveis não paramétricas

${ }^{4}$ Significância estatística estabelecida por meio do Teste U de Mann-Withney

${ }^{5}$ Significância estatística estabelecida pelo teste do Qui-quadrado

de combinações de fármacos imunossupressores de manutenção. O esquema constituído por corticóide, tacrolimus e micofenolato foi utilizado por 14 pacientes $(43,7 \%)$; o esquema composto por corticóide, ciclosporina e azatioprina foi utilizado por sete pacientes $(21,8 \%)$ e o esquema de corticóide, ciclosporina e micofenolato foi utilizado por 11 pacientes (34,3\%). Quatro pacientes (13\%) necessitaram de troca do esquema. Não houve associação significativa entre a presença de placas carotídeas e o tipo de esquema imunossupressor $(\mathrm{p}=0,939)$.

Realizou-se o teste de correlação de Spearman para avaliar a correlação entre o grau de Framingham e a gravidade da placa carotídea, encontrando-se um coeficiente de 0,47 no grupo de pacientes submetidos ao transplante renal $(\mathrm{p}=0,0065)$, o que indica uma correlação intermediária. No grupo dos pacientes em lista de espera para o transplante, encontrou-se um coeficiente de 0,28 ( $\mathrm{p}=0,152)$, ou seja, ausência de correlação. Assim, realizou-se uma análise de regressão logística para fatores associados à presença/ ausência de placa carotídea, a qual está representada na tabela 4 . Encontrou-se que, a cada um ponto de aumento no Escore de Framingham, temos $0,8 \%$ de chance de aumento de placa carotídea, existindo, portanto associação. Não encontramos diferença em relação à presença de placa carotídea quanto ao grupo, diabetes e tempo de diálise. 
Investigação de doença aterosclerótica em pacientes transplantados renais e em lista de espera em acompanhamento ambulatorial por meio de ecografia de artérias carótidas

Tabela 3 - Características presentes no subgrupo dos transplantados renais e persença ou ausência de placas carotídeas

\begin{tabular}{lcccc}
\hline & $\begin{array}{c}\text { Transplante } \\
\text { Renal } \\
(\mathrm{n}=32)\end{array}$ & $\begin{array}{c}\text { Presença } \\
\text { de placa } \\
\text { carotídea }\end{array}$ & $\begin{array}{c}\text { Ausência } \\
\text { de placa } \\
\text { carotídea }\end{array}$ & $\begin{array}{c}\mathrm{p} \\
\text { valor }\end{array}$ \\
\hline $\begin{array}{l}\text { Tempo após } \\
\text { transplante } \\
\text { (meses) }\end{array}$ & $53,34 \pm 59,59$ & $57,79 \pm 59,90$ & $46,85 \pm 60,94$ & $0,399^{3}$ \\
$\begin{array}{l}\text { Doador } \\
\quad \text { Vivo }\end{array}$ & $11(34 \%)$ & $7(63,64 \%)$ & $4(36,36 \%)$ & $0,722^{4}$ \\
\multicolumn{1}{c}{ Falecido } & $21(66 \%)$ & $12(57,14 \%)$ & $9(42,86 \%)$ & \\
$\begin{array}{l}\text { Esquema } \\
\text { Imunossupressor }\end{array}$ & $14(43,7 \%)$ & $8(57,14 \%)$ & $6(42,86 \%)$ & \\
1-CORT+TACRO+MF & & & & \\
2-CORT+CP+AZA & $7(21,8 \%)$ & $4(57,14 \%)$ & $3(42,86 \%)$ & \\
3-CORT+CP+MF & $11(34,3 \%)$ & $7(63,64 \%)$ & $4(36,36 \%)$ & \\
$\begin{array}{l}\text { Troca de esquema } \\
\text { imunossupressor }\end{array}$ & $4(13 \%)$ & & & \\
\hline
\end{tabular}

1- CORT+TACRO+MF: corticoide + tacrolimus + micofenolato

2- CORT+CP+AZA: corticoide + ciclosporina + azatioprina

3- $\mathrm{CORT}+\mathrm{CP}+\mathrm{MF}$ : corticoide + ciclosporina + micofenolato

${ }^{1}$ Os dados são apresentados como número de indivíduos $=\mathrm{n}(\%)$, exceto quando descrito o contrário.

${ }^{2}$ Dados expressos em média \pm desvio padrão.

${ }^{3}$ Significância estatística estabelecida por meio do Teste U de Mann-Withney.

${ }^{4}$ Significância estatística estabelecida pelo teste do Qui-quadrado.

Tabela 4 - Modelo de regressão logística para fatores associados a presença/ausência de placa carotídea

\begin{tabular}{lcccc} 
& & & \multicolumn{2}{c}{ IC OR 95\% } \\
\hline & p valor & OR & Mínimo & Máximo \\
\hline Grupo & 0,271 & 0,415 & 0,087 & 1,988 \\
Framingham & 0,033 & 1,104 & 1,008 & 1,210 \\
Tempo de Diálise & 0,874 & 1,001 & 0,986 & 1,017 \\
Diabetes & 0,076 & 0,222 & 0,042 & 1,171 \\
\hline
\end{tabular}

Qui-quadrado $=16,686$

OR-Odds Ratio

IC- Intervalo de confiança

\section{DISCUSSÃO}

A doença cardiovascular é a principal causa de mortalidade entre pacientes em terapia dialítica e também entre os submetidos ao transplante renal. Uma explicação para tal fato, seria a progressão da doença cardiovascular presente desde o momento do transplanterenal e novos fatores de risco após o transplante, como a terapêutica imunossupressora e o tempo prolongado de diálise prévia. ${ }^{17,18} \mathrm{Em}$ nosso estudo, o grupo submetido ao transplante renal esteve maior tempo em terapia dialítica. Observamos em nossos resultados, que, tanto os pacientes em lista de espera, quanto os já submetidos ao transplante renal, tinham placas carotídeas, com prevalência respectivamente de $70,37 \%$ e de $59,38 \%$. Conforme Freitas et al, a frequência de placas carotídeas em indivíduos com idade média de 63 anos foi de 52\%, que é considerada uma frequência elevada e semelhante à da população adulta mundial. Também relata uma associação da aterosclerose carotídea com idade, obesidade, acidente vascular cerebral e tabagismo. ${ }^{19}$

Em nosso estudo, não encontramos diferença entre os grupos de pacientes quanto à presença de placas carotídeas, porém, o grupo de pacientes que aguardava na lista de espera tinha maior idade, maior prevalência de diabetes, maior escore médio de Framingham e menor tempo em terapia dialítica. Como idade e diabetes, conforme Cofan et al, estão associados a presença de placas carotídeas, ${ }^{5}$ estes podem ter sido fatores confundidores em nosso estudo, contribuindo para não encontrarmos em nossa amostra diferença significativa quanto à presença e gravidade das placas carotídeas.

Akolekar et al, realizaram estudo prospectivo com 1480 pacientes atendidos em ambulatório de avaliação prétransplante renal; destes, 872 permaneceram ativos na lista para receber o transplante. Concluiu-se que a probabilidade de ativação na lista de espera de transplante renal foi menor em pacientes com idade maior ou igual a 65 anos $(\mathrm{p}=0,21)$, tabagistas $(\mathrm{p}<0,0001)$ e diabéticos $(\mathrm{p}=0,004)$. Assim, as características e comorbidades dos pacientes estão associadas à probabilidade de ativação na lista de espera, bem como à realização do transplante renal. ${ }^{20}$ Logo, nosso achado de diferença entre os grupos estudados em relação à idade e à presença de diabetes pode ser explicado pelo fato dos pacientes que aguardam em lista de espera com esse perfil transplantarem menos.

Pádua Netto et al, em estudo que incluiu 126 pacientes transplantados renais sobre estimativa do risco cardiovascular com o escore de Framingham, encontrou 59\% de pacientes com baixo risco de Framingham, 30\% com risco moderado e $11 \%$ com risco elevado. ${ }^{17}$ Observa-se pequena proporção de pacientes com elevado risco cardiovascular por Framingham. Concluiu-se que o risco cardiovascular nos pacientes submetidos ao transplante renal não é completamente explicado pelos fatores de risco tradicionais; assim, o escore de Framingham não quantifica o real risco cardiovascular nessa população. Possivelmente, existem outros fatores de risco para aterosclerose específicos para essa população. ${ }^{17}$ Encontramos resultados semelhantes em nosso estudo, pois a maior parte de nossa amostra de pacientes transplantados renais encontrase distribuída entre as classificações de risco cardiovascular baixa e moderada pelo escore de Framingham. Também constatamos pequena proporção de pacientes com elevado risco cardiovascular.

Ducloux, Kazory e Chalopin avaliaram fatores de risco tradicionais e não tradicionais entre 344 pacientes submetidos a transplante renal sem doença vascular. Framingham demonstrou excelente valor preditivo entre sujeitos de baixo risco, porém subestimou o risco cardiovascular real entre os pacientes de alto risco. ${ }^{21} \mathrm{Em}$ nosso estudo, entre os pacientes submetidos a transplante renal, encontramos uma correlação intermediária entre Framingham e gravidade da placa carotídea.

Já, entre o grupo em lista de espera, não encontramos correlação. 
Polak et al, avaliaram os fatores de risco e as medidas por ecografia de artérias carótidas da população de 6562 membros do Estudo Multi-étnico de Aterosclerose (MESA). Encontraram que, com um seguimento médio de 7,8 anos, todas as placas carotídeas significativas predizem eventos cardiovasculares quando adicionadas aos fatores tradicionais do escore de Framingham. Assim, a aterosclerose carotídea é um marcador de risco cardiovascular na população geral. ${ }^{22}$ Schwaiger et al, realizaram estudo de uma década de seguimento de pacientes candidatos ao transplante renal e demonstraram que a presença de placas carotídeas tem elevado valor preditivo para doença cardiovascular e todas as causas de mortalidade entre os pacientes em hemodiálise. ${ }^{23}$

Em estudo prospectivo com seguimento médio de 9,7 $\pm 2,5$ anos, Cofan et al, avaliaram o impacto da aterosclerose carotídea em 70 pacientes submetidos ao transplante renal por meio de ecodoppler de carótidas. Placas de ateroma foram detectadas em $74 \%$ dos pacientes $(n=52)$. A presença de placas estava associada à idade, hipertensão, diabetes e hipercolesterolemia. As lesões carotídeas foram um fator de risco independente para o óbito de paciente, em longo prazo. Assim, este autor sugere que o ecodoppler de carótidas seja incluído na avaliação de rotina de receptores de transplante renal para identificar pacientes de alto risco. ${ }^{5} \mathrm{Em}$ nosso estudo, encontramos placas carotídeas em 59,38\% dos pacientes, porém nosso estudo foi transversal e nosso tempo médio após transplante foi de 4,44 anos, ou seja, poderíamos ter encontrado neste estudo uma prevalência maior de placas carotídeas se tivéssemos maior tempo médio após o transplante, semelhante ao encontrado no estudo acima citado.

Segundo Boots et al, muitos dos fatores de risco para a doença aterosclerótica em pacientes submetidos ao transplante renal são exacerbados pela terapia imunossupressora, necessária para prevenir a rejeição ao enxerto. ${ }^{7}$ Não encontramos associação significativa entre presença de placas carotídeas e tipo de esquema imunossupressor, porém, segundo Salvador et al, em estudo observacional, o impacto dos imunossupressores na presença de eventos cardiovasculares não está bem estabelecido. ${ }^{24}$ Talvez, essa avaliação não possa ser efetuada por meio de estudos observacionais e necessite ser realizada por meio de ensaios clínicos.

\section{CONCLUSÃO}

A doença aterosclerótica carotídea apresenta prevalência elevada, tanto em pacientes em lista de espera, quanto em pacientes já submetidos ao transplante renal. Os fatores de risco cardiovasculares tradicionais, utilizados no escore de Framingham têm um papel importante no desenvolvimento das placas carotídeas, mas infere-se a possibilidade de outros fatores não tradicionais de risco cardiovascular presentes nessa população envolvidos no desenvolvimento da aterosclerose. A seleção dos pacientes aptos a realizar o transplante renal é bastante rigorosa e a possibilidade de realizá-lo é menor em pacientes com maior idade e com diabetes. Não houve associação significativa entre a presença de placas carotídeas e o tipo de esquema imunossupressor, apesar de estudos na literatura comprovando a exacerbação de fatores de risco cardiovascular com o uso dos mesmos.

Sugerimos a implementação no ambulatório de transplante renal de nosso hospital, a realização de ecografia das artérias carótidas para todos os pacientes em acompanhamento, contribuindo, assim, para o auxílio da estimativa do risco cardiovascular, bem como na determinação da agressividade terapêutica para aterosclerose nesse grupo de pacientes.

\section{ABSTRACT}

Renal transplantation is the treatment of choice for patients with end-stage renal disease. The death proportion related to cardiovascular disease is currently increasing in those patients. Purposes: Assess carotid atherosclerosis prevalence in renal transplanted patients and on the waiting list for the procedure at the Santa Maria University Hospital by carotid arteries ultrasound and determining its correlation to the Framingham score. Methods: Cross-sectional prospective study assessed 59 renal transplanted and non-transplanted patients on the waiting list for renal transplant at the Renal Transplant ambulatory of the Santa Maria Hospital Universitário between January, 2012 and March, 2013. Carotid ultrasound was performed for diagnosis and quantification of carotid artery atherosclerosis, and to calculate the Framingham score. Results: Prevalence of carotid plaques was $59.38 \%$ in patients who underwent renal transplantation, and $70.37 \%$ from those on the waiting list. No significant association was found between groups related to the presence of carotid plaques $(\mathrm{p}=$ $0.379)$ or severity $(\mathrm{p}=0.704)$. The group who underwent renal transplantation has been for longest time on dialysis $(55.25$ \pm 44.16 months vs $28.15 \pm 36.50$ months, $\mathrm{p}=0.00079)$, and presented lower mean age $(45.09 \pm 13.04$ vs. $52.48 \pm 14.18$ years, $\mathrm{p}=0.042)$, fewer diabetic patients $(9 \%$ vs. $52 \%, \mathrm{p}=0.00033)$ and lower Framingham risk score $(8.72 \% \pm 7.5$ vs. $16.51 \% \pm$ $11.97, \mathrm{p}=0.002)$. There was no significant difference between presence of carotid plaque and time of renal transplantation $(\mathrm{p}=0.399)$ or type of immunosuppressive regimen $(\mathrm{p}=0.939)$. Intermediate correlation was found $($ Spearman coeff $=$ $0.47, \mathrm{p}=0.0065$ ) between the Framingham degree and the severity of carotid plaque in patients who underwent renal transplantation. Upon the logistic regression analysis for factors associated to the presence / absence of carotid plaque, an association was found between the Framingham score and the chance of carotid plaque (OR $=1.104$ [1.008 to 1.210, CI OR 95\%], $\mathrm{p}=0.033$ ). Conclusion: Carotid atherosclerotic disease has high prevalence in the population studied. Traditional cardiovascular risk factors used in the Framingham score have an important role in the development of carotid plaques.

Keywords: Renal Transplantation, Atherosclerosis, Carotid Arteries 
Investigação de doença aterosclerótica em pacientes transplantados renais e em lista de espera em acompanhamento ambulatorial por meio de ecografia de artérias carótidas

\section{REFERÊNCIAS}

1. Suthanthiran M, Strom TB. Renal transplantation. N Engl J Med. 1994;331:365-76.

2. ASSOCIAÇÃO BRASILEIRA DE TRANSPLANTES DE ÓRGÃOS. Dimensionamento dos Transplantes no Brasil e em cada estado (2005-2012). Registro Brasileiro de transplantes, veículo oficial da associação brasileira do transplante de órgãos, Ano XVIII, número 4. Disponível em: <http:// www. abto.org.br. htm> Acesso em 5 de dezembro de 2012

3. Ojo AO. Cardiovascular complications after renal transplantation and their prevention. Transplantation. 2006;82:603-11.

4. Cofan F, Nuñez I, Gilabert R, Zambon D, Ros E, Cofan M, et al. Increased prevalence of carotid and femoral atherosclerosis in renal transplant recipients. Transplant Proc. 2001;33:1254-6.

5. Cofan F, Arias M, Nuñez I, Cofan M, Corbella E, Rosich E, et al. Impact of carotid atherosclerosis as assessed by B-mode ultrasonography on the evolution of renal transplantation. Transplant Proc. 2007;39:22368.

6. Halloran PF. Imunossuppre drugs for renal transplantation. N Engl J Med. 2004;351:2715- 29.

7. Boots JMM, Christiaans MH, Van Hooff JP. Effect of Immunosuppressive Agents on Long-Term Survival of Renal Transplant Recipients - Focus on the Cardiovascular Risk. Drugs. 2004;64:2047-73.

8. Dawber TR, Kannel WB. The Framingham Study: an epidemiological approach to coronary heart disease. Circulation. 1966;34:553-5.

9. Sociedade Brasileira de Cardiologia. IV Diretriz Brasileira Sobre Dislipidemias e Prevenção da Aterosclerose Departamento de Aterosclerose da Sociedade Brasileira de Cardiologia. Arquivos Brasileiros de Cardiologia. 2007;88 (Supl. I):2-19

10. D’Agostinho,RBSr, Vasan RS, Pencina MJ, Wolf PA, Cobain M, Massaro JM. et al. General Cardiovascular Risk Profile for Use in Primary Care- The Framingham Heart Study. Circulation. 2008;117:743-53.

11. Campos BAG, Filho WCP. Estenose de Carótida Extracraniana. Arquivos Brasileiros de Cardiologia. 2004;83:528-32.

12. Polak JF, Pencina MJ, Pencina KM, O'Donnell CJ, Wolf PA, D’Agostino R BSr. Carotid-Wall Intima-Media Thickness and Cardiovascular Events. N engl j med. 2011;365:213-21.

13. Jahromi, AS, Cinà, CS, Liu Y, Clase, CM. Sensitivity and specificity of color duplex ultrasound measurement in the estimation of internal carotid artery stenosis: a systematic review and meta-analysis. J Vasc Surg. 2005;41:962-72.
14. National RENAL Foundation. K/DOQI clinical practice guidelines for chronic renal disease: evaluation, classification, and stratification. Am J Renal Dis. 2002;39(2 Suppl 1):S1-266.

15. Grant EG, Benson CB, Moneta GL, Alexandrov AV, Baker JD, Bluth EI, et al. Carotid Artery Stenosis: Gray-Scale and Doppler US Diagnosis-Society of Radiologists in Ultrasound Consensus Conference. Radiology. 2003;229:340-6.

16. Sociedade Brasileira de Cardiologia/ Sociedade Brasileira de Hipertensão / Sociedade Brasileira de Nefrologia. VI Diretrizes Brasileiras de Hipertensão. Arq Bras Cardiol. 2010;95(1 supl.1):1-51.

17. De Pádua Netto MV, Bonfim TC, Costa EN, De Lima HV, Netto LC. Cardiovascular Risk Estimated in Renal Transplant Recipients with the Framingham Score. Transplant Proc. 2012;44:2337-40.

18. Rakhit DJ, Armstrong KA, Beller E, Isbel NM, Marwic, TH. Risk stratification of patients with chronic renal disease: results of screening strategies incorporating clinical risk scoring and dobutamine stress echocardiography. Am Heart J. 2006;152:36370.

19. De Freitas P, Piccinato CE, Martins PW, Filho FM. Aterosclerose carotídea avaliada pelo eco-Doppler: associação com fatores de risco e doenças arteriais sistêmicas. J Vasc Bras. 2008;7:298-307.

20. Akolekar D, Forsythe JLR, Oniscu GC. Impact of patient characteristics and comorbidity profile on activation of patients on the renal transplantation waiting list. Transplant Proc. 2013;45:211522.

21. Ducloux D, Kazory A, Chalopin JM. Predicting coronary heart disease in renal transplant recipients: a prospective study. Renal Int. 2004;66:441-7.

22. Polak JF, Szklo M, Kronmal RA, Burke GL, Shea S, Zavodni AE, et al. The value of Carotid Artery Plaque and Íntima-Média Thickness for Incident Cardiovascular Disease: The Multi-Ethnic Study of Atherosclerosis. J. Am. Heart Assoc. 2013;2:1-10

23. Schwaiger JP, Lamina C, Neyer U, König P, Kathrein H, Sturm W, et al. Carotid Plaques and Their Predictive Value for Cardiovascular Disease and All-Cause Mortality in Hemodialysis Patients Considering Renal Transplantation: A Decade Follow-Up. American Journal of Renal Diseases. 2006;47:888-97.

24. Salvador PF, Pértega-díaz S, Valdés-cañedo F, Seijo-bestilleiro $\mathrm{R}$ Seoane-pillado $\mathrm{T}$, Fernández-rivera $\mathrm{C}$, et al. Incidence of cardiovascular events after renal transplantation and cardiovascular risk scores: study protocol. BMC cardiovascular Disorders. 2011;11:2. 